\title{
Screening Survey of Pain Intensity in Patients with Temporomandibular Disorders
}

\author{
Takashi Uchida1,2, Takashi Iida',3, Masanobu Wakami ${ }^{3}$, Osamu Komiyama',3, Kayo Kuyama ${ }^{4}$ \\ ${ }^{1}$ Orofacial and Head Pain Clinic, Nihon University Hospital at Matsudo, Chiba, Japan \\ ${ }^{2}$ Deparoment of Oral Diagnostics, Nihon University School of Dentistry at Matsudo, Chiba, Japan \\ ${ }^{3}$ Department of Oral Function and Fixed Prosthodontics, Nihon University School of Dentistry at Matsudo, Chiba, Japan \\ ${ }^{4}$ Department of Pathology, Nihon University School of Dentistry at Matsudo, Chiba, Japan \\ Email: uchida.takashi@nihon-u.ac.jp
}

How to cite this paper: Uchida, T., Iida, T., Wakami, M., Komiyama, O. and Kuyama, K. (2021) Screening Survey of Pain Intensity in Patients with Temporomandibular Disorders. Open Journal of Stomatology, 11, 231-243.

https://doi.org/10.4236/ojst.2021.116020

Received: September 2, 2020

Accepted: June 25, 2021

Published: June 28, 2021

Copyright $\odot 2021$ by author(s) and Scientific Research Publishing Inc. This work is licensed under the Creative Commons Attribution International License (CC BY 4.0).

http://creativecommons.org/licenses/by/4.0/

\section{(c) (i) Open Access}

\begin{abstract}
Objective: Pain tends to be the chief complaint in patients suffering temporomandibular disorders (TMD). Previous studies on pain and psychosocial factors have reported on the relationship between presence of pain and mental disorders. To date, however, few studies have addressed the relationship between intensity of pain and psychosocial factors. In this study, we investigated the relationship between intensity of pain and age, gender, palpation scores (PPS), tendencies toward depression, anxiety, and somatization, and oral parafunctional habits. Methods: This screening survey encompassed 104 patients (70 women and 34 men; mean age of $46.1 \pm 19.3$ ) who visited our clinic. We gathered the following data: age; gender; PPS included in Axis I diagnosis; and characteristic pain intensity (CPI), depression, anxiety, somatization, and oral parafunctional habits (assessed by the Oral Behavior Checklist) included in Axis II diagnosis. Based on the results of CPI, we divided patients into two groups: those experiencing low pain intensity (LP group) and those experiencing high pain intensity (HP group). The statistically significant level was set to below 5\%. IBM SPSS Statistics V25 was used to perform all statistical analyses. Results: We observed no gender differences between LP and HP groups. The HP group included significantly more patients with higher scores for depression, anxiety, somatization, and oral parafunctional habits than the LP group. While no gender differences were observed in CPI, depression, anxiety, somatization, and oral parafunctional habits were significantly more common in women than in men. We observed no differences in age or PPS between the LP and HP groups. However, scores for depression, anxiety, somatization, and oral parafunctional habits were significantly higher in the HP group than in the LP group. We performed multiple regression analysis using the CPI score as the dependent variable and scores for depression, anxiety, somatization, and oral parafunctional habits as independent va-
\end{abstract}


riables in both the LP and the HP groups. We identified no significant predictors for the LP group, but extracted depression as a significant predictor in the HP group. On evaluating the correlation of PPS with depression, anxiety, somatization, and oral parafunctional habits in both the LP and the HP groups, we found no correlation between the PPS and the seven-item generalized anxiety disorder (GAD-7) scale in the LP group but identified a significant correlation between the PPS and GAD-7 scores in the HP group. Moreover, the correlation coefficient between the patient health questionnaire (PHQ)-9 and GAD-7 scores was higher in the HP group than in the LP group. Conclusion: In those reporting more intense pain, we found a stronger correlation among psychological factors in patients diagnosed with TMD. Greater tendency toward depression was directly associated with pain intensity. The results point to the need to consider differences in psychosocial factors associated with pain intensity when treating TMD.

\section{Keywords}

Temporomandibular Disorders, DC/TMD, Psychosocial Assessment, Depression

\section{Introduction}

Reports suggest that patients complaining of pain tend to develop psychological disorders [1]. A close correlation between pain and psychosocial disorders has been reported in patients with temporomandibular disorders (TMD) [2]. The Diagnostic Criteria for Temporomandibular Disorders (DC/TMD) recommends combing an Axis I diagnosis for physical assessments and an Axis II diagnosis for psychosocial assessments. Double-sided assessment is necessary for a comprehensive diagnosis of TMD [3].

Variables for Axis II diagnoses included depression, anxiety, somatization, and oral parafunctional habits, as assessed by the Oral Behaviors Checklist (OBC). A number of studies report a relationship between these variables and TMD [4] [5] [6]. Pain tends to be the chief complaint in patients with TMD [7]. Previous studies on pain and psychosocial factors have reported on the relationship between presence of pain and mental disorders [8] [9]. However, few studies to date have addressed the relationship between intensity of pain and psychosocial factors [10].

This study sought to investigate the relationship between intensity of pain and age, gender, palpation scores (PPS; as assessed in Axis I diagnosis of DC/TMD), depression, anxiety, somatization, and oral parafunctional habits (as assessed in Axis II diagnosis of DC/TMD) via a screening survey.

\section{Methods}

\subsection{Patient Sample}

Among patients visiting our clinic with the chief complaint of temporomandi- 
bular dysfunction between October 2019 and March 2021, patients who completed the entire questionnaire, including the PHQ-9, GAD-7, PHQ-15, and $\mathrm{OBC}$, for the Axis II diagnosis of DC/TMD before their medical examination, underwent assessment via DC/TMD examination form (EF) and panoramic radiographic examination. These patients were diagnosed with TMD by a TMD specialist certified by the Japanese Society for Temporomandibular Joint. Finally, 104 patients providing consent to participate (70 women and 34 men with a mean age of $46.1 \pm 19.3$ years) were enrolled in this study, excluding those meeting the following exclusion criteria.

Patients meeting the following criteria were excluded from the study: 1) patients for whom a diagnosis of pain disorder attributable to TMD was ruled out by the DC/TMD protocol; 2) those below 18 years of age; 3 ) those experiencing odontogenic orofacial pain; 4) those with injuries associated with the temporomandibular joint; 5) those with psychiatric disease, those who had visited a neuropsychiatric clinic, or those who had a history of antipsychotic use; 6) those who did not complete the study questionnaire.

The study protocol was approved by the Ethics Committee at the Nihon University School of Dentistry at Matsudo (approval number: EC18-16-11-014-2).

\subsection{Data Collection}

1) Characteristic pain intensity (CPI)

Pain intensity was assessed based on total scores on a graded chronic pain scale and classification grades (three grades: 0, none; 1 - 50, low; 51 - 100, high).

Since all patients enrolled in this study complained of pain, we excluded Grade 0 . They were divided into two groups: those reporting low intensity pain (LP group) and those reporting high intensity pain (HP group).

2) Depression

We assessed depression based on total scores using the Patient Health Questionnaire-9 (PHQ-9) and classification grades (five grades: 0 - 4, none; 5 - 9 , mild; 10 - 14, moderate; 15 - 19, moderate to severe; 20 - 27, severe).

Since none of the patients suffered from severe depression, the patients were classified into four groups for comparison.

3) Anxiety

We assessed anxiety based on total scores for Generalized Anxiety Disorder-7 (GAD-7) items and classification grades (four grades: 0 - 4, none; 5 - 9, mild; 10 - 14, moderate; 15 - 21, severe).

4) Somatization

We assessed somatization (severity of somatic symptoms) based on total scores for the Patient Health Questionnaire-15 (PHQ-15) and classification grades (four grades: 0 - 4, none; 5 - 9, low; 10 - 14, medium; 15 - 30, high).

5) Oral parafunctional habits via $\mathrm{OBC}$

We used total scores obtained using the $\mathrm{OBC}$ and classification grades (three grades: 0 , none; $1-24$, low; $25-84$, high) to assess oral parafunctional habits.

Since all patients in the study reported oral parafunctional habits, we excluded 
Grade 0. Patients were grouped into low or high grades for comparison.

\subsection{Statistical Analysis}

We applied the chi-square test to compare gender differences and grade distribution of oral parafunctional habits, and Fisher's exact test to compare distribution for depression, anxiety, somatization between the LP and HP groups.

We applied the Mann-Whitney $U$ test to analyze male and female differences in CPI scores, PPS, and scores for depression, anxiety, somatization and oral parafunctional habits and to analyze inter-group differences in age, PPS, and scores for depression, anxiety, somatization and oral parafunctional habits.

We performed multiple regression analysis using the CPI score as the dependent variable and depression, anxiety, somatization, and oral parafunctional habits as independent variables in both the LP and the HP groups.

We calculated Spearman's rank correlation coefficient between depression, anxiety, and somatization and oral parafunctional habits in both the LP and the HP groups. We then evaluated the significance of the difference in the correlation coefficient between the LP and the HP groups using Fisher's transformation from $r$ to $z$ [11].

A p-value $<0.05$ was deemed statistically significant. All statistical analyses were performed with IBM SPSS Statistics V25 (IBM Corporation).

\section{Results}

\subsection{Grades of Study Variables According to Pain Intensity} (Table 1)

We found no significant differences for gender in the grades for study variables between the LP and HP groups.

According to the grade, patients graded "none" accounted for the highest proportion of patients in the LP group. That is, we found fewer patients classified as mild, moderate, or moderate to severe for depression, mild, moderate, or severe for anxiety, and low, medium, or high for somatization than numbers of patients graded "none". However, in the HP group, the proportion of patients complaining of depression, anxiety, or somatization was significantly greater than patients graded "none". Grades for oral parafunctional habits were higher in the HP group than in the LP group. Grades for oral parafunctional habits were higher in the HP group than in the LP group $(p<0.05)$.

\subsection{Gender (Table 2)}

No significant gender differences were observed in CPI scores. Scores for depression, anxiety, somatization, and oral parafunctional habits were significantly higher in women than in men $(p<0.01)$.

\subsection{Scores for Study Variables According to Pain Intensity (Table 3)}

We observed no differences in age or PPS between the LP and HP groups. How- 
ever, scores for depression, anxiety, somatization, and oral parafunctional habits were significantly higher in the HP group than in the LP group $(p<0.01)$.

\subsection{Predictors According to Pain Intensity (Table 4)}

While no significant predictors were identified in the LP group, depression was extracted as a significant predictor in the HP group $(p<0.05)$.

\subsection{Correlation Coefficient between Study Variables Depending on the Intensity of Pain (Table 5, Table 6)}

We observed a significant correlation between the PPS and the study variables, except between the PPS and GAD-7 in the LP group and between the PPS and $\mathrm{OBC}$ in the HP group. While we found no correlation between the PPS and GAD-7 scores in the LP group $(r=0.252)$, we observed moderate correlation between them in the HP group $(r=0.505)$. In contrast, we found a moderate and

Table 1. Grade of subjects studied by differences in pain intensity.

\begin{tabular}{|c|c|c|c|c|}
\hline & Number of & Low Intensity & High Intensity & DU \\
\hline & Patients & $(\mathrm{n}=53)$ & $(\mathrm{n}=51)$ & \\
\hline Gender & & & & 0.264 \\
\hline Female & 70 & 33 & 37 & \\
\hline Male & 34 & 20 & 14 & \\
\hline Depression (PHQ-9 Grade) & & & & $0.003^{* *}$ \\
\hline None & 54 & 36 & 18 & \\
\hline Mild & 33 & 13 & 20 & \\
\hline Moderate & 11 & 4 & 7 & \\
\hline Moderate-to-Severe & 5 & 0 & 5 & \\
\hline Severe & 1 & 0 & 1 & \\
\hline Anxiety (GAD-7 Grade) & & & & $0.006^{* *}$ \\
\hline None & 67 & 42 & 25 & \\
\hline Mild & 22 & 8 & 14 & \\
\hline Moderate & 13 & 3 & 10 & \\
\hline Severe & 2 & 0 & 2 & \\
\hline Somatization (PHQ-15 Grade) & & & & $0.015^{*}$ \\
\hline None & 50 & 33 & 17 & \\
\hline Low & 35 & 15 & 20 & \\
\hline Medium & 13 & 4 & 9 & \\
\hline High & 6 & 1 & 5 & \\
\hline Oral Behaviors Checklist (OBC Grade) & & & & $0.003^{* *}$ \\
\hline Low & 77 & 46 & 31 & \\
\hline High & 27 & 7 & 20 & \\
\hline
\end{tabular}

${ }^{*}: p<0.05,{ }^{* *}: p<0.01$. 
Table 2. Consideration by gender.

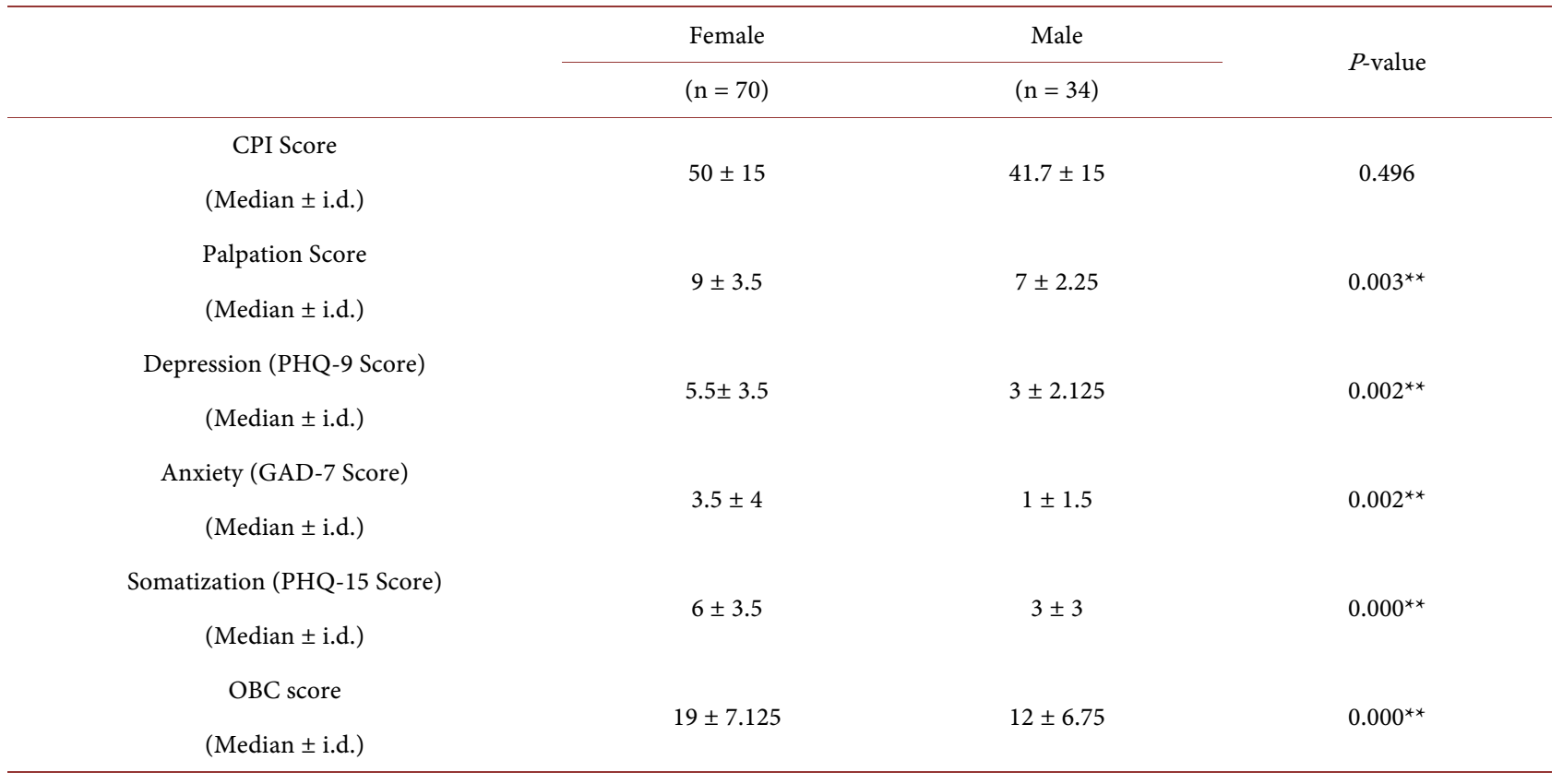

${ }^{* *}: p<0.01$.

Table 3. Scores of subjects studied by differences in pain intensity.

\begin{tabular}{|c|c|c|c|}
\hline & Low intensity & High intensity & \multirow{2}{*}{$P$-value } \\
\hline & $(\mathrm{n}=53)$ & $(\mathrm{n}=51)$ & \\
\hline Age & \multirow[b]{2}{*}{$40 \pm 21.25$} & \multirow[b]{2}{*}{$46 \pm 12.5$} & \multirow[b]{2}{*}{0.695} \\
\hline (Mdion $+i d$ ) & & & \\
\hline Palpation Score & \multirow{3}{*}{$8 \pm 3.5$} & \multirow{3}{*}{$8 \pm 4$} & \multirow{3}{*}{0.114} \\
\hline & & & \\
\hline (Median \pm i.d.) & & & \\
\hline Depression (PHQ-9 Score) & \multirow[b]{2}{*}{$3 \pm 2.5$} & \multirow[b]{2}{*}{$7 \pm 3.5$} & \multirow[b]{2}{*}{$0.001^{* *}$} \\
\hline (Median \pm i.d.) & & & \\
\hline Anxiety (GAD-7 Score) & \multirow[b]{2}{*}{$1 \pm 2$} & \multirow[b]{2}{*}{$5 \pm 4$} & \multirow[b]{2}{*}{$0.003^{* *}$} \\
\hline (Median $+i d)$ & & & \\
\hline Somatization (PHQ-15 Score) & \multirow{3}{*}{$3 \pm 2$} & \multirow{3}{*}{$7 \pm 3.5$} & \multirow{3}{*}{$0.004^{* x}$} \\
\hline & & & \\
\hline (Median \pm i.d.) & & & \\
\hline OBC score & \multirow[b]{2}{*}{$15 \pm 6$} & \multirow[b]{2}{*}{$21 \pm 8.5$} & \multirow[b]{2}{*}{$0.007^{\star *}$} \\
\hline (Median \pm i.d.) & & & \\
\hline
\end{tabular}

${ }^{* *}: p<0.01$.

Table 4. Predictors of differences in pain intensity.

\begin{tabular}{|c|c|c|c|c|c|c|c|c|c|c|}
\hline \multirow{3}{*}{$\begin{array}{c}\text { Variables } \\
\text { Depression }\end{array}$} & \multicolumn{5}{|c|}{ Low intensity $(\mathrm{n}=53)$} & \multicolumn{5}{|c|}{ High Intensity $(n=51)$} \\
\hline & B & $\begin{array}{l}\text { Standard } \\
\text { Error (SE) }\end{array}$ & & & P-Value & B & $\begin{array}{l}\text { Standard } \\
\text { Error (SE) }\end{array}$ & & & $P$-value \\
\hline & -0.98 & 0.785 & -2.558 & 0.597 & 0.218 & 1.419 & 0.623 & 0.165 & 2.674 & $0.027^{\star}$ \\
\hline
\end{tabular}




\section{Continued}

\begin{tabular}{|c|c|c|c|c|c|c|c|c|c|c|}
\hline \multicolumn{11}{|l|}{ (PHQ-9 Score) } \\
\hline $\begin{array}{c}\text { Anxiety } \\
\text { (GAD-7 Score) }\end{array}$ & 1.006 & 0.795 & -0.592 & 2.604 & 0.212 & -0.975 & 0.583 & -2.148 & 0.198 & 0.101 \\
\hline $\begin{array}{l}\text { Somatization } \\
\text { (PHQ-15 Score) }\end{array}$ & -0.321 & 0.584 & -0.873 & 1.514 & 0.582 & 0.408 & 0.467 & -0.532 & 1.349 & 0.387 \\
\hline OBC score & 0.066 & 0.226 & -0.388 & 0.52 & 0.77 & -0.005 & 0.118 & -0.383 & 0.373 & 0.978 \\
\hline
\end{tabular}

Table 5. Correlation coefficient of investigated variables in group low intensity (GL) and group High intensity (GH).

\begin{tabular}{|c|c|c|c|c|c|c|c|c|c|c|}
\hline & \multicolumn{5}{|c|}{ Low intensity $(n=53)$} & \multicolumn{5}{|c|}{ High intensity $(\mathrm{n}=51)$} \\
\hline & PPS & PHQ-9 & GAD-7 & PHQ-15 & OBC & PPS & PHQ-9 & GAD-7 & PHQ-15 & OBC \\
\hline Palpation Score (PPS) & 1 & & & & & 1 & & & & \\
\hline Depression (PHQ-9 Score) & $0.515^{* *}$ & 1 & & & & $0.586^{* *}$ & 1 & & & \\
\hline Anxiety (GAD-7 Score) & 0.252 & $0.599^{* *}$ & 1 & & & $0.505^{* *}$ & $0.817^{* *}$ & 1 & & \\
\hline Somatization (PHQ-15 Score) & $0.436^{* *}$ & $0.585^{* *}$ & $0.597^{* *}$ & 1 & & $0.617^{* *}$ & $0.690^{* *}$ & $0.586^{* *}$ & 1 & \\
\hline OBC score & $0.419^{* *}$ & $0.469^{* *}$ & $0.341^{*}$ & $0.378^{* *}$ & 1 & 0.267 & $0.343^{*}$ & $0.332^{*}$ & $0.415^{* *}$ & 1 \\
\hline
\end{tabular}

*: $p<0.05,{ }^{* *}: p<0.01$.

Table 6. Comparison of the correlations between group low intensity (GL) and group High intensity (GH).

\begin{tabular}{|c|c|c|c|c|c|c|}
\hline & & & Low intensity & High intensity & & \\
\hline & & & $(\mathrm{n}=53)$ & $(\mathrm{n}=51)$ & & $P$ \\
\hline \multirow[t]{4}{*}{ PPS } & $:$ & PHQ-9 & $\mathrm{r}=0.515 ; p<0.01$ & $\mathrm{r}=0.586 ; p<0.01$ & -0.5 & 0.617 \\
\hline & $:$ & GAD-7 & $\mathrm{r}=0.252 ; \mathrm{ns}$ & $\mathrm{r}=0.505 ; p<0.01$ & -1.48 & 0.139 \\
\hline & $:$ & PHQ-15 & $\mathrm{r}=0.436 ; p<0.01$ & $\mathrm{r}=0.617 ; p<0.01$ & -1.25 & 0.211 \\
\hline & $:$ & $\mathrm{OBC}$ & $\mathrm{r}=0.419 ; \mathrm{g}<0.01$ & $\mathrm{r}=0.267 ; \mathrm{ns}$ & 0.86 & 0.39 \\
\hline \multirow[t]{3}{*}{ PHQ-9 } & $:$ & GAD-7 & $\mathrm{r}=0.599 ; p<0.01$ & $\mathrm{r}=0.817 ; p<0.01$ & -2.26 & $0.024^{*}$ \\
\hline & $:$ & PHQ-15 & $\mathrm{r}=0.585 ; p<0.01$ & $\mathrm{r}=0.690 ; p<0.01$ & -0.88 & 0.379 \\
\hline & $:$ & $\mathrm{OBC}$ & $\mathrm{r}=0.469 ; p<0.01$ & $\mathrm{r}=0.343 ; p<0.05$ & 0.75 & 0.423 \\
\hline \multirow[t]{2}{*}{ GAD-7 } & $:$ & PHQ-15 & $\mathrm{r}=0.597 ; \mathrm{g}<0.01$ & $\mathrm{r}=0.586 ; p<0.01$ & 0.08 & 0.936 \\
\hline & $:$ & OBC & $\mathrm{r}=0.341 ; p<0.05$ & $\mathrm{r}=0.332 ; p<0.05$ & 0.05 & 0.96 \\
\hline PHQ-15 & $:$ & OBC & $\mathrm{r}=0.378 ; p<0.01$ & $\mathrm{r}=0.415 ; p<0.01$ & -0.2 & 0.842 \\
\hline
\end{tabular}

${ }^{*}: p<0.05,{ }^{* *}: p<0.01$.

statistically significant correlation between PPS and PHQ-15 results in the LP group $(r=0.419)$; this correlation was not observed in the HP group $(r=0.267)$. The correlation between the PHQ-9 and GAD-7 scores was moderate $(r=0.599)$ in the LP group but strong $(r=0.817)$ in the HP group, indicating a significantly higher correlation in the HL group than in the LP group $(p<0.05)$. 


\section{Discussion}

A previous study of psychosocial factors in patients with TMD suggested scores for psychosocial factors such as depression, anxiety, and somatization are higher in patients with TMD than in healthy individuals [12]. This study involved a screening survey focusing on pain intensity in patients with TMD only. No healthy controls were involved in the study. Figure 1 illustrates the correlation between study variables.

Gender is considered a risk factor for TMD. Female adolescents are likely to be at increased risk of pain due to TMD [13], whereas the intensity of pain reportedly declines with increasing age in women [14]. The mean age of the participants in this study was $46.7 \pm 19.3$ years. The study excluded those below 18 years of age. Due to the small sample size of the adolescent participants, it is possible there is no correlation between pain intensity and gender differences. However, since psychosocial characteristics observed in women, including tendencies toward depression, anxiety, and somatization significantly influence the prevalence of TMD [15], we investigated the relationship between gender differences and study variables other than age. We found that PPS, depression, anxiety, somatization, and oral parafunctional habits were all significantly higher in women than in men. Gender differences in patient psychosocial factors have been observed with TMD [16]. Specifically, the neurophysiological mechanisms underlying the relationship between pain and depression appear to be more complex in women than in men [17]. Male and female differences in sensitivity to pain appear to exist [18]. Moreover, women are more likely to complain of psychological distress and psychosocial stress and to recognize somatization than men [19] and are significantly more likely to report depression and anxiety [20]. Thus, gender differences appear to influence psychosocial factors. The results of this study indicate that while there may be no direct relationship between gender difference and pain intensity in individuals with TMD, women are more likely to report increased OBC and PPS with increasing psychosocial factors and oral parafunctional habits than men, suggesting that gender differences are indirectly related to pain intensity.

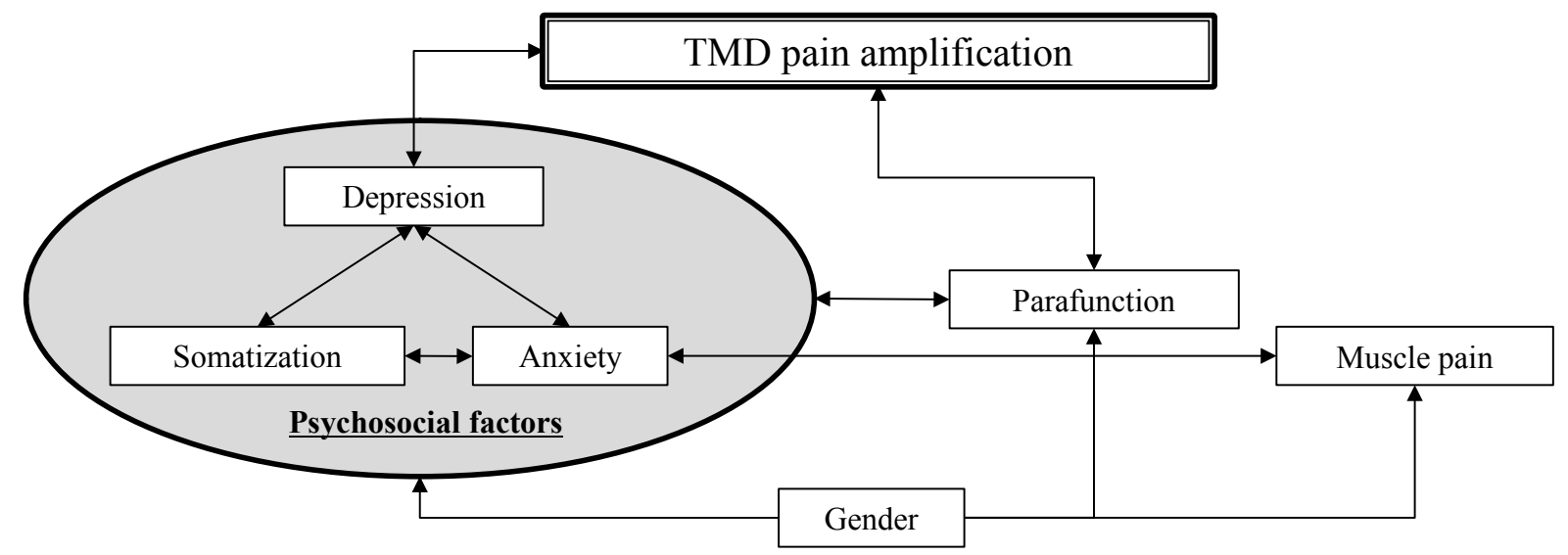

Figure 1. Correlation diagram of the investigated variables. 
Psychological disorders are commonly observed in patients with TMD [21]. Higher scores for depression, anxiety, and somatization are all reported in patients with TMD than in the general population [22]. Numerous studies have reported on depression, anxiety, and somatization as psychosocial factors in patients with TMD. Patients with TMD complicated by pain are highly likely to develop depression and somatization [22], and the incidence of depression and somatization is associated with pain [23]. There is a report stating that anxiety is a less significant psychosocial factor in patients with TMD [24]. Simon et al. [4] reported, however, that patients with TMD are more likely to experience tendencies toward depression and anxiety than the general population, and assert that there is a strong correlation between TMD and depression and anxiety, and that screening is essential via the PHQ-9 and GAD-7 in the examination of TMD. Thus, the significance of anxiety, as well as depression is reported, and it is pointed out that there is a high correlation between patients with TMD and psychosocial disorders [2].

In this study, the HP group for the grade distribution of depression, anxiety, and somatization showed a tendency for significantly more severe disorders than the LP group. The scores for depression, anxiety, somatization, and oral parafunctional habits were also significantly higher in the HP group than in the LP group, confirming that psychosocial factors vary depending on the intensity of pain. Thus, we performed multiple regression analysis using the CPI score as the dependent variable in both the LP and the HP groups. The results revealed that significant predictors were not identified in the LP group, but depression was extracted as a significant predictor in the HP group, and thus, depression is the most influential factor for the intensity of pain. Ascertaining the relationship of TMD with depression is important [25]. There is a high association of depression in patients with TMD complicated by pain [9]. Patients with TMD who experience a higher tendency toward depression are likely to have a negative perception of their health condition, indicating a positive correlation between CPI scores and a tendency for depression. Thus, the perception of their health condition becomes bad with increasing CPI scores [26]. Another study reported that pain in patients with TMD is a trigger to cause or worsen a tendency for depression [27]. However, it is difficult to determine whether pain associated with TMD causes changes in psychosocial factors or vice versa [28]. This study was also unable to determine the directional relationship between psychosocial factors and pain; however, the relationship between depression and pain intensity was stronger with increasing intensity of pain, although this relationship was not observed in patients for whom the intensity of pain was low. Specifically, changes in psychosocial conditions varied depending on the intensity of pain in patients with TMD.

We also calculated correlation coefficients between study variables depending on the intensity of pain. The results revealed that there are generally moderate correlations between various variables, confirming the mutual correlation of psychosocial factors such as depression, anxiety, and somatization in both the LP 
and the HP groups. PPS, which is recognized as an indicator for muscle pain, was not correlated with GAD-7 scores in the LP group, but moderately correlated with it in the HP group. Anxiety is an important psychosocial factor that influences pain in patients with TMD [20], and it is positively correlated with awake bruxism [10]. Anxiety is also shown to be associated with muscle pain as a risk indicator for myofascial pain [29]. This study also confirmed that there is a correlation between high PPS and muscle symptoms associated with changes in anxiety. However, there was no correlation between PPS and PHQ-15 results in the HP group. This may be because somatization is not related to the number of palpation sites but to an increase in the intensity of pain, although increasing tendencies toward depression and anxiety are also associated with a tendency toward somatization. In future examination of PPS for a diagnosis of TMD, it would be preferable to determine not just the number of palpation sites, but the intensity of pain [30]. In addition, the correlation coefficient between the PHQ-9 and GAD-7 scores was significantly higher with increasing intensity of pain. While no relationship between tendencies toward depression and anxiety has been established in patients with TMD [20], one study reports a strong correlation between scores for the PHQ-9 and for the GAD-7 [31], suggesting that a closer correlation between depression and anxiety is associated with higher intensity of pain; thus, the correlation between psychological factors varies with increasing intensity of pain.

In this study, we selected PPS, which can be quantified as a study variable, from variables included in the Axis I diagnosis of DC/TMD to assess the correlation between pain intensity and Axis I diagnosis. We found no significant difference in PPS between the LP and HP groups. However, PPS correlated with depression and anxiety in both the LP and the HP groups. Psychosocial factors and the results of a palpation test in Axis I diagnosis were positively correlated with TMD symptoms [30]. However, another study gives contrary results, finding no positive correlation between Axis I and II diagnoses [32]. Views on the correlation between PPS and Axis I and II diagnoses vary from researcher to researcher and such correlation is controversial. In this study, PPS correlated with study variables in Axis II diagnosis, suggesting a correlation between the number of palpation sites in Axis I diagnosis and psychosocial factors in Axis II diagnosis.

Evaluating psychosocial factors for the diagnosis of TMD is essential to identifying the risk factors of onset and continuance of TMD [28]. However, pain associated with TMD and psychosocial factors is more complex than pain associated with symptoms such as depression, anxiety, and somatization [8]. Based on the results of our present study, rather than considering whether the correlation of TMD with individual depression, anxiety, and somatization affects pain, we should regard the three psychosocial disorders as mutually correlated and consider a tendency toward depression to be a representative factor influencing pain. We propose that the mutual correlation between the three psychosocial disorders increases with more intense pain and that the influence of TMD on the 
intensity of pain also increases.

\section{Conclusion}

TMD was not directly related to PPS for Axis I diagnosis of DC/TMD in terms of pain intensity, but related to psychological factors for Axis II diagnosis. We confirmed that the relationship between individual psychosocial factors is greater with increasing intensity of pain in patients with TMD and that a greater tendency toward depression is directly related to the intensity of pain. In treating patients with TMD, it may be necessary to consider changes in psychosocial factors based on the intensity of the reported pain.

\section{Conflicts of Interest}

The authors declare no conflicts of interest regarding the publication of this paper.

\section{References}

[1] Keefe, F.J., Rumble, M.E., Scipio, C.D., Giordano, L.A. and Perri, L.M. (2004) Psychological Aspects of Persistent Pain: Current State of the Science. The Journal of Pain, 5, 195-211. https://doi.org/10.1016/j.jpain.2004.02.576

[2] Manfredini, D., Marini, M., Pavan, C., Pavan, L. and Guarda-Nardini, L. (2009) Psychosocial Profiles of Painful TMD Patients. Journal of Oral Rehabilitation, 36, 193-198. https://doi.org/10.1111/j.1365-2842.2008.01926.x

[3] Schiffman, E. and Ohrbach, R. (2016) Executive Summary of the Diagnostic Criteria for Temporomandibular Disorders for Clinical and Research Applications. The Journal of the American Dental Association, 147, 438-445. https://doi.org/10.1016/j.adaj.2016.01.007

[4] Simoen, L., Van den Berghe, L., Jacquet, W. and Marks, L. (2020) Depression and Anxiety Levels in Patients with Temporomandibular Disorders: Comparison with the General Population. Clinical Oral Investigations, 24, 3939-3945. https://doi.org/10.1007/s00784-020-03260-1

[5] Gonzalez, Y.M., Nickel, J.C., Scott, J.M., Liu, H. and Iwasaki, L.R. (2018) Psychosocial Scores and Jaw Muscle Activity in Women. Journal of Oral Facial Pain and Headache, 32, 381-388. https://doi.org/10.11607/ofph.2133

[6] Chow, J.C. and Cioffi, I. (2019) Effects of Trait Anxiety, Somatosensory Amplification, and Facial Pain on Self-Reported Oral Behaviors. Clinical Oral Investigations, 23, 1653-1661. https://doi.org/10.1007/s00784-018-2600-1

[7] Laskin, D.M., Greene, C.S. and Hylander, W.L. (2006) Temporomandibular Disorders: An Evidence-Based Approach to Diagnosis and Treatment. Quintessence, Chicago.

[8] Giannakopoulos, N.N., Keller, L., Rammelsberg, P., Kronmüller, K.T. and Schmitter, M. (2010) Anxiety and Depression in Patients with Chronic Temporomandibular Pain and in Controls. Journal of Dentistry, 38, 369-376. https://doi.org/10.1016/j.jdent.2010.01.003

[9] Osiewicz, M., Lobbezoo, F., Ciapała, B., Pytko-Polończyk, J. and Manfredini, D. (2020) Pain Predictors in a Population of Temporomandibular Disorders Patients. Journal of Clinical Medicine, 9, 452. https://doi.org/10.3390/jcm9020452

[10] Su, N., Lobbezoo, F., van Wijk, A., van der Heijden, G.J. and Visscher, C.M. (2017) 
Associations of Pain Intensity and Pain-Related Disability with Psychological and Socio-Demographic Factors in Patients with Temporomandibular Disorders: A Cross-Sectional Study at a Specialised Dental Clinic. Journal of Oral Rehabilitation, 44, 187-196. https://doi.org/10.1111/joor.12479

[11] Przystańska, A., Jasielska, A., Ziarko, M., Pobudek-Radzikowska, M., Maciejewska-Szaniec, Z., et al. (2019) Psychosocial Predictors of Bruxism. BioMed Research International, 2019, Article ID: 2069716. https://doi.org/10.1155/2019/2069716

[12] Lajnert, V., Francisković, T., Grzic, R., Pavicić, D.K., Bakarbić, D., et al. (2010) Depression, Somatization and Anxiety in Female Patients with Temporomandibular Disorders (TMD). Collegium Antropologicum, 34, 1415-1419.

[13] Pereira. L.J., Pereira-Cenci, T., Pereira, S.M., Cury, A.A., Ambrosano, G.M., et al. (2009) Psychological Factors and the Incidence of Temporomandibular Disorders in Early Adolescence. Brazilian Oral Research, 23, 155-160. https://doi.org/10.1590/S1806-83242009000200011

[14] Komiyama, O., Obara, R., Iida, T., Nishimura, H., Okubo, M., et al. (2014) Age-Related Associations between Psychological Characteristics and Pain Intensity among Japanese Patients with Temporomandibular Disorder. Journal of Oral Science, 56, 221-225. https://doi.org/10.2334/josnusd.56.221

[15] Licini, F., Nojelli, A., Segù, M. and Collesano, V. (2009) Role of Psychosocial Factors in the Etiology of Temporomandibular Disorders: Relevance of a Biaxial Diagnosis. Minerva Stomatologica, 58, 557-566.

[16] Levitt, S.R. and McKinney, M.W. (1994) Validating the TMJ Scale in a National Sample of 10,000 Patients: Demographic and Epidemiologic Characteristics. Journal of Orofacial Pain, 8, 25-35.

[17] Miettinen, O., Lahti, S. and Sipilä, K. (2012) Psychosocial Aspects of Temporomandibular Disorders and Oral Health-Related Quality-of-Life. Acta Odontologica Scandinavica, 70, 331-336. https://doi.org/10.3109/00016357.2011.654241

[18] Dao, T.T. and LeResche, L. (2000) Gender Differences in Pain. Journal of Orofacial Pain, 14, 169-184.

[19] Fillingim, R.B., Ohrbach, R., Greenspan, J.D., Knott, C., Diatchenko, L., et al. (2013) Psychological Factors Associated with Development of TMD: The OPPERA Prospective Cohort Study. The Journal of Pain, 14, T75-T90. https://doi.org/10.1016/j.jpain.2013.06.009

[20] Chuinsiri, N. and Jitprasertwong, P. (2020) Prevalence of Self-Reported Pain-Related Temporomandibular Disorders and Association with Psychological Distress in a Dental Clinic Setting. Journal of International Medical Research, 48. https://doi.org/10.1177/0300060520951744

[21] Bonjardim, L.R., Gavião, M.B., Pereira, L.J. and Castelo, P.M. (2005) Anxiety and Depression in Adolescents and Their Relationship with Signs and Symptoms of Temporomandibular Disorders. International Journal of Prosthodontics, 18, 347-352.

[22] Canales, G.T., Bonjardim, L.R., Poluha, R.L., Fonseca, F., Soares, C., et al. (2020) Correlation between Physical and Psychosocial Findings in a Population of Temporomandibular Disorder Patients. International Journal of Prosthodontics, 33, 155-159. https://doi.org/10.11607/ijp.5847

[23] Yap. A.U., Tan, K.B., Chua, E.K. and Tan, H.H. (2002) Depression and Somatization in Patients with Temporomandibular Disorders. The Journal of Prosthetic Dentistry, 88, 479-484. https://doi.org/10.1067/mpr.2002.129375

[24] Reiter, S., Emodi-Perlman, A., Goldsmith, C., Friedman-Rubin, P. and Winocur, E. (2015) Comorbidity between Depression and Anxiety in Patients with Temporo- 
mandibular Disorders According to the Research Diagnostic Criteria for Temporomandibular Disorders. Journal of Oral \& Facial Pain and Headache, 29, 135-143. https://doi.org/10.11607/ofph.1297

[25] Yap, A.U., Chua, E.K., Tan, K.B. and Can, Y.H. (2004) Relationships between Depression/Somatization and Self-Reports of Pain and Disability. Journal of Orofacial Pain, 18, 220-225.

[26] Blanco-Hungría, A., Rodríguez-Torronteras, A., Blanco-Aguilera, A., Biedma-Velázquez, L., Serrano-Del-Rosal, R., et al. (2012) Influence of Sociodemographic Factors upon Pain Intensity in Patients with Temporomandibular Joint Disorders Seen in the Primary Care Setting. Medicina Oral, Patologia Oral, Cirugia Bucal, 17, e1034-1041. https://doi.org/10.4317/medoral.17576

[27] Yeung, E., Abou-Foul, A., Matcham, F., Poate, T. and Fan, K. (2017) Integration of Mental Health Screening in the Management of Patients with Temporomandibular Disorders. British Journal of Oral and Maxillofacial Surgery, 55, 594-599. https://doi.org/10.1016/j.bjoms.2017.03.014

[28] Fillingim, R.B., Ohrbach, R., Greenspan, J.D., Knott, C., Dubner, R., et al. (2011) Potential Psychosocial Risk Factors for Chronic TMD: Descriptive Data and Empirically Identified Domains from the OPPERA Case-Control Study. Journal of Pain, 12, 46-60. https://doi.org/10.1016/j.jpain.2011.08.007

[29] Kindler, S., Samietz, S., Houshmand, M., Grabe, H.J., Bernhardt, O., et al. (2012) Depressive and Anxiety Symptoms as Risk Factors for Temporomandibular Joint Pain: A Prospective Cohort Study in the General Population. Journal of Pain, 13, 1188-1197. https://doi.org/10.1016/j.jpain.2012.09.004

[30] Almoznino, G., Zini, A., Zakuto, A., Zlutzky, H., Bekker, S., et al. (2019) Muscle Tenderness Score in Temporomandibular Disorders Patients: A Case-Control Study. Journal of Oral Rehabilitation, 46, 209-218. https://doi.org/10.1111/joor.12743

[31] Yap, A.U. and Natu, V.P. (2020) Inter-Relationships between Pain-Related Temporomandibular Disorders, Somatic and Psychological Symptoms in Asian Youths. Journal of Oral Rehabilitation, 47, 1077-1083. https://doi.org/10.1111/joor.13033

[32] Canales, G.T., Guarda-Nardini, L, Rizzatti-Barbosa, C.M., Conti, P.C.R. and Manfredini, D. (2019) Distribution of Depression, Somatization and Pain-related Impairment in Patients with Chronic Temporomandibular Disorders. Journal of Applied Oral Science, 27, e20180210. https://doi.org/10.1590/1678-7757-2018-0210 